\title{
ON A CLASS OF TRUTH-VALUE EVALUATIONS OF THE PRIMITIVE LOGIC
}

\author{
KATUZI ONO
}

\section{Introduction}

Main purpose of the present paper is to exhibit a class of truth-value evaluations of the primitive logic $\boldsymbol{L O}$ and its sentential part $\boldsymbol{L O S}^{1)}$.

$\boldsymbol{L O}$ is the logic having two logical constants, IMPLICATION $\rightarrow$ and UNIVERSAL QUANTIFICATION ( ), and the following inference rules:

$\boldsymbol{F}: \quad \mathfrak{A}$ is deducible from $\mathfrak{A}$.

$\boldsymbol{I}: \mathfrak{B}$ is deducible from $\mathfrak{A}$ and $\mathfrak{A} \rightarrow \mathfrak{B}$.

$\boldsymbol{I}^{*}: \mathfrak{A} \rightarrow \mathfrak{B}$ is deducible from the fact that $\mathfrak{B}$ is deducible from $\mathfrak{A}$.

$\boldsymbol{U}: \mathfrak{A}(t)$ is deducible from $(x) \mathfrak{A}(x)$.

$\boldsymbol{U}^{*}: \quad(x) \mathfrak{A}(x)$ is deducible from the fact that $\mathfrak{A}(t)$ is deducible for any variable $t$ whatever.

LOS is the logic having the sole logical constant IMPLICATION $\rightarrow$ and the inference rules $\boldsymbol{F}, \boldsymbol{I}$, and $\boldsymbol{I}^{*}$ only.

The domain of truth-values can be regarded as the value-domain of an evaluation $\boldsymbol{E}$ which associates to every proposition its truth-value. Let $\boldsymbol{B}$ be the class of propositions. Then, any proposition variable $p$ can be regarded as a variable running over $\boldsymbol{B}$, and its evaluation $p^{*}$ can be regarded as a variable running over the domain $\boldsymbol{D}$ of truth-values. To logical constants, we associate combinations or operations which are so defined that $\boldsymbol{E}$ is a homomorphism of $\boldsymbol{B}$ into $\boldsymbol{D}$. Any mapping $\boldsymbol{E}$ ( $\boldsymbol{B}$ into $\boldsymbol{D})$ of this kind is called a SEMI-EVALUATION of the logic. The homomorphic image of a proposition $\mathfrak{p}$ by $\boldsymbol{E}$ is called the $\boldsymbol{E}$-image of $\mathfrak{p}$ and denoted by $\boldsymbol{E}(\mathfrak{p})$ or simply by $\mathfrak{p}^{*}$. In the following, I will denote the $\boldsymbol{E}$-images of logical constants by the same notations as the original logical constants unless there is a fear of ambiguity.

Received August 29, 1966.

1) See Ono [1]. 
Any semi-evaluation $\boldsymbol{E}$ of a logic is called an EVALUATION of the logic if and only if $\boldsymbol{E}(\mathfrak{p})=0$ holds identically whenever $\mathfrak{p}$ is a proposition provable in the logic.

In (1), I will prove a few theorems concerning evaluations of LOS and $\boldsymbol{L} \boldsymbol{O}$.

Theorem 1 gives a sufficient condition for that a semi-evaluation of $\boldsymbol{L O S}$ is an evaluation of it. The condition is

E 1: $p^{*} \rightarrow 0=0$,

$\boldsymbol{E} 2: \quad p^{*} \rightarrow p^{*}=0$,

E 3: $0 \rightarrow p^{*}=p^{*}$,

E 4: $p^{*} \rightarrow\left(p^{*} \rightarrow q^{*}\right)=p^{*} \rightarrow q^{*}$,

E 5: $p^{*} \rightarrow\left(q^{*} \rightarrow r^{*}\right)=q^{*} \rightarrow\left(p^{*} \rightarrow r^{*}\right)$,

E 6: $p^{*} \rightarrow q^{*}=0$ implies $\left(r^{*} \rightarrow p^{*}\right) \rightarrow\left(r^{*} \rightarrow q^{*}\right)=0$.

Theorem 2 is a theorem corresponding to Theorem 1 for the logic $\boldsymbol{L O}$.

Theorem 3 shows a way to construct an evaluation out of a class of evaluations satisfying the conditions in Theorem 1 or in Theorem 2.

ExAmples $1-4$ of (2) give two extreme classes of evaluations of LOS and LO . THEOrem 3 indicates a way of constructing a class of evaluations lying between them.

A sufficient condition for that any proposition $\mathfrak{p}$ satisfying $\boldsymbol{E}(\mathfrak{p})=0$ identically is identically true in the ordinary two-valued logic is given in Theorem 4 (for $\boldsymbol{L O S}$ ) and in Theorem 5 (for $\boldsymbol{L O}$ ). As well known, any proposition of the ordinary two-valued sentential logic is provable in the classical sentential logic, so Theorem 4 gives a sufficient condition for that any proposition $\mathfrak{p}$ of a sentential logic satisfying $\boldsymbol{E}(\mathfrak{p})=0$ is provable in the classical logic. The condition is only that the domain of the evaluation $\boldsymbol{E}$ has at least two members.

In (2), I will give some examples of evaluations of $\boldsymbol{L O S}$ and $\boldsymbol{L O}$ together with some remarks about them.

In the present paper, I adopt the practical way $\boldsymbol{P D}$ for describing formal deductions introduced in my paper [2] . Concerning proof-notes, I will use the nomenclature introduced in my paper [3]. 


\section{(1) Theorems on evaluations of the primitive logic.}

THeOrem 1. Let $\boldsymbol{E}$ be any semi-evaluation of the logic LOS satisfying identically the following conditions:

$\boldsymbol{E} 1: p^{*} \rightarrow 0=0$,

$\boldsymbol{E} 2: p^{*} \rightarrow p^{*}=0$,

$\boldsymbol{E} 3: 0 \rightarrow p^{*}=p^{*}$,

E 4: $p^{*} \rightarrow\left(p^{*} \rightarrow q^{*}\right)=p^{*} \rightarrow q^{*}$,

$\boldsymbol{E} 5: \quad p^{*} \rightarrow\left(q^{*} \rightarrow r^{*}\right)=q^{*} \rightarrow\left(p^{*} \rightarrow r^{*}\right)$,

E 6: $\quad p^{*} \rightarrow q^{*}=0$ implies $\left(r^{*} \rightarrow p^{*}\right) \rightarrow\left(r^{*} \rightarrow q^{*}\right)=0$.

Then, $\boldsymbol{E}$ is an evaluation of $\mathbf{L O S}$.

Proof. Let $\Pi$ be any proof-note in $\boldsymbol{L O S}$ arranged in the fundamental order of steps ${ }^{2}$, and $\underline{s}$ be any step in $\Pi$. Let $\mathfrak{p}$ be any provable proposition of the step $\underline{s}$ and $\left\{\mathfrak{p}_{1}, \ldots, \mathfrak{p}_{n}\right\}$ be the whole set of propositions of assumption steps of $\underline{s}$. Further, let $\mathfrak{q}_{1}, \ldots, \mathfrak{q}_{n}$ be any sequence of propositional variables. Then, I assert that the $\boldsymbol{E}$-image of $\mathfrak{q}_{1} \rightarrow\left(\mathfrak{q}_{2} \rightarrow\left(\ldots \rightarrow\left(\mathfrak{q}_{n} \rightarrow \mathfrak{p}\right) \ldots\right)\right.$ (this proposition is called the proposition associated to the step $\underline{s}$ and is denoted simply by $\mathfrak{q}_{1}, \ldots, \mathfrak{q}_{n} \rightarrow \mathfrak{p}$ from now on') is identically equal to 0 as far as $\mathfrak{q}_{1}^{*} \rightarrow \mathfrak{p}_{1}^{*}=0, \ldots, \mathfrak{q}_{n}^{*} \rightarrow \mathfrak{p}_{n}^{*}=0$.

I will prove this assertion by complete induction as it holds true for the first step of $\Pi$ because the first step must be an assumption step of itself and this assertion holds true for any step having the same proposition as its assumption step by virtue of $\boldsymbol{E} \mathbf{1}, \boldsymbol{E} \mathbf{2}$, and $\boldsymbol{E} \mathbf{5}$. So, I will prove that this assertion holds true for any step $\underline{\boldsymbol{s}}$ of $\Pi$ as far as it holds true for every step of $\Pi$ II standing before $\underline{\boldsymbol{s}}$. If $\underline{\boldsymbol{s}}$ is an assumption step of itself, the assertion holds true as has been remarked above.

If $\underline{\boldsymbol{s}}$ is a deduced step, it must be deduced by the inference rule $\boldsymbol{F}$ (Case $\boldsymbol{F})$, deduced by the inference rule $\boldsymbol{I}$ (Case $\boldsymbol{I}$ ), or deduced by the inference rule $\boldsymbol{I}^{*}\left(\right.$ Case $\left.\boldsymbol{I}^{*}\right)$.

Case $\boldsymbol{F}$, where $\underline{\boldsymbol{s}}$ is deduced from a step $\underline{\boldsymbol{u}}$ by the inference rule $\boldsymbol{F}$ : The set

2) Any step of the form $\underline{\boldsymbol{r}} \boldsymbol{A}$ is called an assumption step of $\underline{\boldsymbol{s}}$ if and only if $\underline{\boldsymbol{s}}$ can be expressed in the form $\underline{\boldsymbol{r}} \boldsymbol{u} \boldsymbol{w}$. If we normalize the lengths of index-words occurring in a proof-note by adjoining suitable numbers of $\diamond$ 's at their ends and arrange the steps of the proof-note according to the lexicographic order of their normalized index-words regarding $\diamond$ as the last letter, we have the fundamental order of steps. As for details, see ONo [3]. 
$\left\{\mathfrak{p}_{1}, \ldots, \mathfrak{p}_{n}\right\}$ of the propositions of assumption steps of $\underline{s}$ includes the set of the propositions of assumption steps of $\underline{\boldsymbol{u}}$. Hence, by virtue of $\boldsymbol{E} \mathbf{1}, \boldsymbol{E} \mathbf{2}$, and $\boldsymbol{E} 5$, the expression $\mathfrak{q}_{1}^{*}, \ldots, \mathfrak{q}_{n}^{*} \rightarrow \mathfrak{p}^{*}$ is identically equal to 0 as far as $\mathfrak{q}_{i}^{*} \rightarrow \mathfrak{p}_{i}^{*}=0$ for $i=1, \ldots, n$.

Case $\boldsymbol{I}$, where $\underline{\boldsymbol{s}}$ is deduced from the steps $\underline{\boldsymbol{u}}$ and $\underline{\boldsymbol{v}}$ : Let the propositions of $\underline{\boldsymbol{u}}$ and $\underline{\boldsymbol{v}}$ be $\mathfrak{h}$ and $\mathfrak{h} \rightarrow \mathfrak{t}$, respectively. Then, the proposition of $\underline{\boldsymbol{s}}$ is $\mathfrak{t}$. Now, the set $\left\{\mathfrak{p}_{1}, \ldots, \mathfrak{p}_{n}\right\}$ includes the set of the propositions of assumption steps of $\underline{\boldsymbol{u}}$ as well as that of $\underline{\boldsymbol{v}}$. Accordingly, for any specification of truthvalues of variables satisfying $\mathfrak{q}_{i}^{*} \rightarrow \mathfrak{p}_{i}^{*}=0$ for every $i=1, \ldots, n$, the formulas $\mathfrak{q}_{1}^{*}, \ldots, \mathfrak{q}_{n}^{*} \rightarrow \mathfrak{h}^{*}=0$ and $\mathfrak{q}_{1}^{*}, \ldots, \mathfrak{q}_{n}^{*} \rightarrow\left(\mathfrak{h}^{*} \rightarrow \mathfrak{f}^{*}\right)=0$ hold by virtue of our induction assumption, $\boldsymbol{E} \mathbf{4}$, and $\boldsymbol{E} \mathbf{5}$. The latter implies $\left(\mathfrak{q}_{1}^{*}, \ldots, \mathfrak{q}_{n}^{*} \rightarrow \mathfrak{h}^{*}\right) \rightarrow$ $\left(q_{1}^{*}, \ldots, q_{n}^{*} \rightarrow \mathfrak{l}^{*}\right)=0$ by virtue of $\boldsymbol{E} \mathbf{4}, \boldsymbol{E} \mathbf{5}$, and $\boldsymbol{E} \mathbf{6}$. Hence, $0 \rightarrow$ $\left(\mathfrak{q}_{1}^{*}, \ldots, \mathfrak{q}_{n}^{*} \rightarrow \mathfrak{f}^{*}\right)$ must be equal to 0 for this specification. On the other hand, this must be equal to $\mathfrak{q}_{1}^{*}, \ldots, \mathfrak{q}_{n}^{*} \rightarrow \mathfrak{f}^{*}$ according to $\boldsymbol{E} 3$. Consequently, $\mathfrak{q}_{1}^{*}, \ldots, \mathfrak{q}_{n}^{*} \rightarrow \mathfrak{l}^{*}=0$ holds for this specification.

Case $\boldsymbol{I}^{*}$, where $\boldsymbol{s}$ is deduced from the fact that the step $\boldsymbol{s} \epsilon$ is deducible from the step $\underline{\boldsymbol{s}} \boldsymbol{A}$ : Let the propositions of $\underline{\boldsymbol{A}}$ and $\underline{\boldsymbol{s}} \in$ be $\mathfrak{h}$ and $\mathfrak{f}$, respectively. Then, the proposition of $\underline{s}$ is $\mathfrak{h} \rightarrow \mathfrak{f}$. The propositions associated to $\underline{\boldsymbol{s}} \boldsymbol{\epsilon}$ and $\underline{s}$ are of the forms

$$
\mathfrak{q}_{1}, \ldots, \mathfrak{q}_{n}, \mathfrak{q}_{n+1} \rightarrow \mathfrak{f} \text { and } \mathfrak{q}_{1}, \ldots, \mathfrak{q}_{n} \rightarrow(\mathfrak{h} \rightarrow \mathfrak{l}) \text {, }
$$

respectively. The $\boldsymbol{E}$-image of the former is identically equal to 0 as far as $\mathfrak{q}_{1}^{*} \rightarrow \mathfrak{p}_{1}^{*}=0, \ldots, \mathfrak{q}_{n}^{*} \rightarrow \mathfrak{p}_{n}^{*}=0$, and $\mathfrak{q}_{n+1}^{*} \rightarrow \mathfrak{h}^{*}=0$. By taking $\mathfrak{q}_{n+1}^{*}=\mathfrak{h}^{*}$ and taking $\boldsymbol{E} 2$ into consideration, we can see that

$$
\mathfrak{q}_{1}^{*}, \ldots, \mathfrak{q}_{n}^{*}, \mathfrak{h}^{*} \rightarrow \mathfrak{l}^{*}=0 \text { i.e. } \mathfrak{q}_{1}^{*}, \ldots, \mathfrak{q}_{n}^{*} \rightarrow\left(\mathfrak{h}^{*} \rightarrow \mathfrak{l}^{*}\right)=0
$$

holds as far as $q_{1}^{*} \rightarrow \mathfrak{p}_{1}^{*}=0, \ldots, q_{n}^{*} \rightarrow \mathfrak{p}_{n}^{*}=0$ hold.

Theorem 2. Let $\boldsymbol{E}$ be any semi-evaluation of the logic LO satisfying identically the following conditions:

E 1: $p^{*} \rightarrow 0=0$,

E 2: $p^{*} \rightarrow p^{*}=0$,

$\boldsymbol{E} 3: \quad 0 \rightarrow p^{*}=p^{*}$,

E 4: $p^{*} \rightarrow\left(p^{*} \rightarrow q^{*}\right)=p^{*} \rightarrow q^{*}$,

E 5: $p^{*} \rightarrow\left(q^{*} \rightarrow r^{*}\right)=q^{*} \rightarrow\left(p^{*} \rightarrow r^{*}\right)$,

E 6: $p^{*} \rightarrow q^{*}=0$ implies $\left(r^{*} \rightarrow p^{*}\right) \rightarrow\left(r^{*} \rightarrow q^{*}\right)=0$, 
E 7: $(x) p^{*}(x) \rightarrow p^{*}(t)=0$,

E 8: If $u^{*} \rightarrow p^{*}(t)=0$ for every $t$, then $u^{*} \rightarrow(x) p^{*}(x)=0$.

Then, $\boldsymbol{E}$ is an evaluation of $\boldsymbol{L O}$.

Proof. Let $\Pi$ be any proof-note of $\boldsymbol{L} \boldsymbol{O}$ arranged in the fundamental order of steps, and $\underline{s}$ be any step in $\Pi$. Let $\mathfrak{p}$ be the proposition of the step $\underline{s}$, and $\left\{\mathfrak{p}_{1}, \ldots, \mathfrak{p}_{n}\right\}$ be the whole set of propositions of the assumption steps of $\underline{s}$ skipping over denomination steps ${ }^{3}$.

Let $\mathfrak{q}_{1}, \ldots, q_{n}$ be any sequence of proposition variables. Then, I can assert as in the proof of the preceding theorem that the $\boldsymbol{E}$-image of the proposition $\mathfrak{q}_{1}, \ldots, \mathfrak{q}_{n} \rightarrow \mathfrak{p}$ associated to the step $\underline{\mathbf{s}}$ is identically equal to 0 as far as $\mathfrak{q}_{1}^{*} \rightarrow \mathfrak{p}_{1}^{*}=0, \ldots, \mathfrak{q}_{n}^{*} \rightarrow \mathfrak{p}_{n}^{*}=0$. This assertion can be proved also by complete induction, since this assertion can be proved easily for any assumption step $\underline{s}$. Accordingly, let us assume that the assertion holds true for any step of II standing before $\boldsymbol{s}$. If $\underline{s}$ is a deduced step, it must be deduced by the inference rule $\boldsymbol{F}, \boldsymbol{I}$, or $\boldsymbol{I}^{*}$ (Case $\boldsymbol{F}$, Case $\boldsymbol{I}$, or Case $\boldsymbol{I}^{*}$, respectively), or it is deduced by the inference rules $\boldsymbol{U}$ or $\boldsymbol{U}^{*}$ (Case $\boldsymbol{U}$ or Case $\boldsymbol{U}^{*}$, respectively). In Cases $\boldsymbol{F}, \boldsymbol{I}$, or $\boldsymbol{I}^{*}$, we can prove our assertion for the step $\boldsymbol{s}$ quite similarly as we have proved the corresponding facts in the proof of the preceding theorem. So, I will prove our assertion here only in the Cases $\boldsymbol{U}$ and $\boldsymbol{U}^{*}$.

Case $\boldsymbol{U}$, where the step $\underline{\boldsymbol{s}}$ is deduced from a step $\underline{\boldsymbol{r}}$ by the inference rule $\boldsymbol{U}$ : The propositions of the steps $\underline{\boldsymbol{r}}$ and $\underline{\boldsymbol{s}}$ are of the forms $(x) \mathfrak{p}(x)$ and $\mathfrak{p}(t)$. Because any assumption step of $\underline{\boldsymbol{r}}$ is also an assumption step of $\underline{\boldsymbol{s}}$, $\mathfrak{q}_{1}^{*}, \ldots, \mathfrak{q}_{n}^{*} \rightarrow(x) \mathfrak{p}^{*}(x)$ must be identically equal to 0 as far as $\mathfrak{q}_{1}^{*} \rightarrow \mathfrak{p}_{1}^{*}=0$, $\ldots, \mathfrak{q}_{n}^{*} \rightarrow \mathfrak{p}_{n}^{*}=0$ according to $\boldsymbol{E} \mathbf{1}, \boldsymbol{E} \mathbf{4}$, and $\boldsymbol{E} \mathbf{5}$. On the other hand,

$$
\left(\mathfrak{q}_{1}^{*}, \ldots, \mathfrak{q}_{n}^{*} \rightarrow(x) \mathfrak{p}^{*}(x)\right) \rightarrow\left(\mathfrak{q}_{1}^{*}, \ldots, \mathfrak{q}_{n}^{*} \rightarrow \mathfrak{p}^{*}(t)\right)=0
$$

by virtue of $\boldsymbol{E} \mathbf{6}$ and $\boldsymbol{E} 7$, so $0 \rightarrow\left(\mathfrak{q}_{1}^{*}, \ldots, \mathfrak{q}_{n}^{*} \rightarrow \mathfrak{p}^{*}(t)\right)=0$, therefore $\mathfrak{q}_{1}^{*}, \ldots, \mathfrak{q}_{n}^{*} \rightarrow \mathfrak{p}^{*}(t)=0$, according to $\boldsymbol{E} 3$, holds true as far as $\mathfrak{q}_{1}^{*} \rightarrow \mathfrak{p}_{1}^{*}=0$, $\ldots, \mathfrak{q}_{n}^{*} \rightarrow \mathfrak{p}_{n}^{*}=0$.

Case $\boldsymbol{U}^{*}$, where the step $\underline{\boldsymbol{s}}$ is deducible from the fact that $\underline{\boldsymbol{s}} \epsilon$ is deducible from the denomination $\boldsymbol{s} \boldsymbol{A}$ of the form $\forall t$ !, i.e. from the fact that $\underline{\boldsymbol{s}} \boldsymbol{\epsilon}$ is deducible for any

3) In proof-notes of $\boldsymbol{L O}$, some steps are denominations of the form $\forall \boldsymbol{t}$ !. These steps are skipped over in the present proof. 
variable $t$ whatever. The propositions of the steps $\underline{s}$ and $\underline{\boldsymbol{s}} \epsilon$ are propositions of the forms $(x) \mathfrak{p}(x)$ and $\mathfrak{p}(t)$, respectively. Because any assumption step of $\underline{\boldsymbol{s}} \epsilon$ except for the denomination $\underline{\boldsymbol{s}} \boldsymbol{A}$ is also an assumption step of $\underline{\boldsymbol{s}}$, we can well assume according to $\boldsymbol{E} \mathbf{1}, \boldsymbol{E} 4$, and $\boldsymbol{E} 5$ that $\mathfrak{q}_{1}^{*}, \ldots, \mathfrak{q}_{n}^{*} \rightarrow \mathfrak{p}^{*}(t)=0$ holds for any variable $t$ whatever as far as $\mathfrak{q}_{1}^{*} \rightarrow \mathfrak{p}_{1}^{*}=0, \ldots, \mathfrak{q}_{n}^{*} \rightarrow \mathfrak{p}_{n}^{*}=0$. Consequently, $\mathfrak{q}_{1}^{*}, \ldots, \mathfrak{q}_{n}^{*} \rightarrow(x) \mathfrak{p}^{*}(x)$ must be identically equal to 0 as far as $\mathfrak{q}_{1}^{*} \rightarrow \mathfrak{p}_{1}^{*}=0, \ldots, \mathfrak{q}_{n}^{*} \rightarrow \mathfrak{p}_{n}^{*}=0$, according to $\boldsymbol{E} 8$.

Theorem 3. Let $\left\{\boldsymbol{E}_{i} ; i \in \boldsymbol{J}\right\}$ be any family of evaluations of LOS (or LO) satisfying $\boldsymbol{E} \mathbf{1}-\boldsymbol{E} 6$ (or $\boldsymbol{E} \mathbf{1}-\boldsymbol{E} 8$ ) identically. Let $\boldsymbol{D}_{i}$ be the domain of truth values of the evaluation $\boldsymbol{E}_{i}$, and 0 of $\boldsymbol{D}_{i}$ be denoted by $0_{i}$. I will denote the $\boldsymbol{E}_{i}$-image of $\mathfrak{p}$ by $\boldsymbol{E}_{i}(\mathfrak{p})$ or $\mathfrak{p}^{i}$ and the $\boldsymbol{E}_{i}$-image of $\rightarrow$ and () by $\stackrel{i}{\rightarrow}$ and ()$^{i}$, respectively. Let us now define a new semi-evaluation $\boldsymbol{E}$ as follows:

1) The domain $\boldsymbol{D}$ of truth-values of $\boldsymbol{E}$ is formed by functions $p^{*}$ defined over $\boldsymbol{J}$ satisfying $p^{*}(i)=\boldsymbol{D}_{\imath}$,

2) 0 of $\boldsymbol{D}$ is defined by $0(i)=0_{i}$,

3) $p^{*}(i)=p^{i}$,

4) $\left(p^{*} \rightarrow q^{*}\right)(i)=p^{i} \stackrel{i}{\rightarrow} q^{i}$,

5) $\left((x) p^{*}(x)\right)(i)=(x)^{i} p^{i}(x) \quad($ for $\boldsymbol{L O}$ only $)$.

Then, $\boldsymbol{E}$ is an evaluation of $\mathbf{L O S}$ (or LO).

Proof. It can be proved without difficulty that $\boldsymbol{E} \mathbf{1}-\boldsymbol{E} \mathbf{6}$ (or $\boldsymbol{E} \mathbf{1}-\boldsymbol{E} 8$ ) hold for the semi-evaluation $\boldsymbol{E}$. Hence, $\boldsymbol{E}$ is an evaluation of $\boldsymbol{L O S}$ (or $\boldsymbol{L O}$ ) according to Theorem 1 (or Theorem 2).

THeOREM 4. Let $\boldsymbol{E}$ be any evaluation of LOS satisfying $\boldsymbol{E} \mathbf{1}-\boldsymbol{E} \mathbf{6}$, and let us assume that the domain $\boldsymbol{D}$ of truth-values of $\boldsymbol{E}$ has at least two members. Then, any proposition whose $\boldsymbol{E}$-image is identically equal to 0 is provable in the classical sentential logic.

Proof. In the domain $\boldsymbol{D}$, there are 0 and a member other than 0 because $\boldsymbol{D}$ is assumed to have at least two members. The member of $\boldsymbol{D}$ other than 0 is denoted by 1 . Then, by virtue of $\boldsymbol{E} \mathbf{1}, \boldsymbol{E} \mathbf{2}$, and $\boldsymbol{E} \mathbf{3}$, we can prove

$$
0 \rightarrow 0=1 \rightarrow 0=1 \rightarrow 1=0 \text { and } 0 \rightarrow 1=1 .
$$


Namely, the combination $\rightarrow$ behaves just as the IMPLICATION of the ordinary two valued logic with respect to the pair $\{0,1\}$ of truth-values. It is also remarkable that $\{0,1\}$ is closed with respect to the combination $\rightarrow$.

Let $\mathfrak{p}$ be any proposition whose $\boldsymbol{E}$-image $\mathfrak{p}^{*}$ is identically equal to 0 . Then, $\mathfrak{p}$ is also identically equal to 0 for any specification of variables which specify them to 0 or 1 . Accordingly, $\mathfrak{p}^{*}$ is identically equal to 0 with respect to the ordinary evaluation of two-valued sentential logic. Because the ordinary two-valued sentential logic is nothing but the classical sentential logic, so $\mathfrak{p}$ must be provable in the classical sentential logic.

Theorem 5. Let $\boldsymbol{E}$ be any evaluation of $\boldsymbol{L O}$ satisfying $\boldsymbol{E} \mathbf{1}-\boldsymbol{E} 8$, and let us assume that the domain $\boldsymbol{D}$ of truth-values of $\boldsymbol{E}$ has a member 1 such that

1) $1 \neq 0$,

2) $1 \rightarrow u^{*}=0$ implies $u^{*} \rightarrow 1=0$ or $u^{*}=0$.

Then, any proposition whose $\boldsymbol{E}$-image is identically equal to 0 is also identically true in the usual two-valued logic.

Proof. Let $\mathfrak{p}$ be any proposition whose $\boldsymbol{E}$-image is identically equal to 0 with respect to an evaluation $\boldsymbol{E}$ satisfying the above mentioned condition. Let us take up the sub-domain $\boldsymbol{D}_{0}=\left\{u^{*} ; 1 \rightarrow u^{*}=0\right\}$. Then, clearly $0,1 \epsilon \boldsymbol{D}_{0}$. Moreover, $\boldsymbol{D}_{0}$ is closed with respect to the logical operations $\rightarrow$ and ( ). Hence, we have an evaluation $\boldsymbol{E}_{0}$ of $\boldsymbol{L} \boldsymbol{O}$ satisfying $\boldsymbol{E} \mathbf{1}-\boldsymbol{E} \mathbf{8}$ by restricting its value domain to $\boldsymbol{D}_{0}$. In the domain $\boldsymbol{D}_{0}$, we can replace every member of $\boldsymbol{D}_{0}$ other than 0 by 1 keeping $\boldsymbol{E} \mathbf{1}-\boldsymbol{E} 8$ and $\boldsymbol{p}^{*}=0$ identically true. Hence, $\boldsymbol{p}^{*}=0$ holds identically for the usual evaluation of the two valued logic.

\section{(2) Examples and remarks.}

Remark 1. The domain $\boldsymbol{D}$ of truth-values satisfying the conditions $\boldsymbol{E} \mathbf{1}-\boldsymbol{E} \mathbf{6}$ or $\boldsymbol{E} \mathbf{1}-\boldsymbol{E} \mathbf{8}$ is almost a partly ordered system with the minimum member 0 with respect to the ordering $p \geq q$ defined by $p \rightarrow q=0$. This relation $\geq$ is really reflexive and transitive, but we can not assert that $p \geq q$ and $q \geq p$ imply $p=q$. Naturally, $p \geq 0$ is always true, and more over, we can deduce $p=0$ from $0 \geq p$, for this relation $\geq$.

Example 1. Let $\boldsymbol{D}$ be any semi-lattice having for any pair of its 
members the union of the pair and satisfying the following condition: For any pair of members $p^{*}$ and $q^{*}$ of $\boldsymbol{D}$, there exists a member $u^{*}$ satisfying

1) $p^{*} \cup u^{*} \geq q^{*}$,

2) For any $w^{*}, p^{*} \cup w^{*} \geq q^{*}$ implies $w^{*} \geq u^{*}$.

The member $u^{*}$ uniquely determined by $p^{*}$ and $q^{*}$ is denoted by $p^{*} \rightarrow q^{*}$.

The member $p^{*} \rightarrow p^{*}$ of $\boldsymbol{D}$ can be proved to be independent of $p^{*}$. It is proved to be the minimum member of $\boldsymbol{D}$, so $I$ will denote it by 0 . With respect to the special member 0 and the combination $\rightarrow$, the conditions $\boldsymbol{E} \mathbf{1}-\boldsymbol{E} \mathbf{6}$ can be proved to hold. So, we can define an evaluation $\boldsymbol{E}_{s}$ of $\boldsymbol{L O S}$ by making use of the domain $\boldsymbol{D}$.

Example 2. Let $\boldsymbol{V}$ be any domain of objects and let $\boldsymbol{D}$ be any semilattice having for any number of its members the union of them and satisfying the condition for defining the combination $\rightarrow$ in ExAmpLE 1 . We deal with functions of any number of variables running over $\boldsymbol{V}$ and having $\boldsymbol{D}$ as their value domain. Composite functional expressions can be constructed starting from expressions of the form $f^{*}(x, \ldots, z)$ (elementary formulas) by the combination $\rightarrow$ and the operators of the form $(x)$ which stands for $\underset{x \in \dot{v}}{U} \quad$ Just as in Example 1, we can prove that $\boldsymbol{D}$ has its minimum member 0 . With respect to this minimum member 0 , the combination $\rightarrow$, and the operators of the form $(x)$, the conditions $\boldsymbol{E} 1-\boldsymbol{E} 8$ hold true. So, we can define an evaluation $\boldsymbol{E}_{p}$ of $\boldsymbol{L} \boldsymbol{O}$ by making use of $\boldsymbol{D}$.

ExAmple 3. Let $\boldsymbol{D}$ be any partly ordered system having the minimum member 0 . Then, we can define two operators $\underline{0}$ and $\underline{1}$ over $\boldsymbol{D}$ by $\underline{\mathbf{0}} \cdot p^{*}=0$ and $\mathbf{1} \cdot p^{*}=p^{*}$. We can further define two-variable function $\boldsymbol{X}$ over $\boldsymbol{D}$ by that $\boldsymbol{X}\left(p^{*}, q^{*}\right)$ is the operator $\underline{\boldsymbol{0}}$ if $p^{*} \geq q^{*}$ and it is the operator $\underline{\mathbf{1}}$ otherwise. The value domain of $\boldsymbol{X}$ is the pair-set of the operators $\underline{0}$ and 1. By making use of this function $\boldsymbol{X}$, I will define a combination $\rightarrow$ of members of $\boldsymbol{D}$ by $p^{*} \rightarrow q^{*}=\boldsymbol{X}\left(p^{*}, q^{*}\right) \cdot q^{*}$. By this combination $\rightarrow$, we obtain a member of $\boldsymbol{D}$ from any ordered pair of members of $\boldsymbol{D}$. With respect to the minimum member 0 and the combination $\rightarrow$, the conditions $\boldsymbol{E} \mathbf{1}-\boldsymbol{E} \mathbf{6}$ hold. So, we can define an evaluation $\boldsymbol{E}^{\boldsymbol{s}}$ of $\boldsymbol{L O S}$ by making use of $\boldsymbol{D}$.

Example 4. Let $\boldsymbol{V}$ be any domain of objects and let $\boldsymbol{D}$ be any semilattice having for any number of its members the union of them. We deal 
with functions of any number of variables running over $\boldsymbol{V}$ and having $\boldsymbol{D}$ as their value-domain. We can define the combination $\rightarrow$ over $\boldsymbol{D}$ just as we have done in ExAmple 3. By this combination $\rightarrow$, we obtain a member of $\boldsymbol{D}$ for any ordered pair of members of $\boldsymbol{D}$. Composite functional expressions can be constructed starting from expressions of the form $f^{*}(x, \ldots, z)$ (elementary formulas) by the combination $\rightarrow$ and the operators of the form (x) which stands for $\cup_{x \in V}$ As the union of nullset (a subset of $\boldsymbol{D}$ ) must be the minimum member of $\boldsymbol{D}$, the domain surely has its minimum member 0 . With respect to the minimum member 0 , the combination $\rightarrow$, and the operators of the form $(x)$, the conditions $\boldsymbol{E} \mathbf{1}-\boldsymbol{E} \mathbf{8}$ hold true. So, we can define an evaluation $\boldsymbol{E}^{p}$ of $\boldsymbol{L} \boldsymbol{O}$ by making use of $\boldsymbol{D}$.

Remark 2. Example 1 (or Example 2) and Example 3 (or Éxample 4) show two extremities of evaluations of $\boldsymbol{L O S}$ (or $\boldsymbol{L O}$ ). To show the difference, let us take $\boldsymbol{D}$ as the class of positive (including 0 ) valued functions $p^{*}, q^{*}, \ldots$ of a variable $x$ running over the closed interval $[0,1]$. We regard here $p^{*} \geq q^{*}$ as denoting $(x)\left(p^{*}(x) \geq q^{*}(x)\right)$, the range of the quantification variable $x$ is $[0,1]$. Take for example $p^{*}(x)=x$ and $q^{*}(x)=1-x$. Let $\rightarrow$ and $\rightarrow$ be the $\boldsymbol{E}_{s}$-image (or $\boldsymbol{E}_{p}$-image) and $\boldsymbol{E}^{s}$-image (or $\boldsymbol{E}^{p}$-image) of $\rightarrow$ of $\boldsymbol{L O S}$ (or $\boldsymbol{L O}$ ). Then,

$$
\begin{aligned}
& \left(p^{*} \rightarrow q^{*}\right)(x)=1-x \quad \text { in } \quad 0 \leq x \leq \frac{1}{2} \\
& =0 \quad \text { in } \quad \frac{1}{2}<x \leq 1 .
\end{aligned}
$$

On the other hand,

$$
\left(p^{*} \rightarrow q^{*}\right)(x)=1-x \text { everywhere in }[0,1] .
$$

Intermediate evaluations can be constructed by dividing $[0,1]$ into a number of intervals, defining $\boldsymbol{E}_{s}$ (or $\boldsymbol{E}_{p}$ ) for every intervals, and combining these evaluations by making use of Theorem 3 . For example, let $\boldsymbol{E}^{*}$ be the combined evaluations of the $\boldsymbol{E}_{s^{-}}$or $\boldsymbol{E}_{p}$-evaluations for the intervals $\left[0, \frac{1}{3}\right],\left(-\frac{1}{3}, \frac{2}{3}\right)$, and $\left[\frac{2}{3}, 1\right]$. Then,

$$
\begin{aligned}
\left(p^{*} \rightarrow q^{*}\right)(x) & =1-x & & \text { in } & & 0 \leq x<\frac{2}{3} \\
& =0 & & \text { in } & & \frac{2}{3} \leq x \leq 1,
\end{aligned}
$$


where $\rightarrow$ is the $\boldsymbol{E}^{*}$-image of $\rightarrow$ of $\boldsymbol{L O S}$ (or $\boldsymbol{L O}$ ). Evidently, $\boldsymbol{E}^{*}$-image of any proposition lies between $\boldsymbol{E}_{s^{-}}$and $\boldsymbol{E}^{s}$-images (or $\boldsymbol{E}_{p^{-}}$and $\boldsymbol{E}^{p}$-images) of the same proposition.

\section{REFERENCES}

[1] Ono, K., On universal character of the primitive logic, Nagoya Math. J., 27-1 (1966), 311-353.

[2] - On a practical way of describing formal deductions, Nagoya Math. J., 21 (1962), $115-121$.

[3] - A study on formal deductions in the primitive logic Nagoya Math. J., 31 (1967), $1-14$. 